\section{AB0295 CLINICAL CHARACTERISTICS OF 31 CASES OF MALIGNANT SOLID TUMORS IN PATIENTS WITH RHEUMATOID ARTHRITIS}

Akiko Aoki ${ }^{1}$, Hiroshi Kobayashi ${ }^{2}$, Hiroshi Oka ${ }^{3} .{ }^{1}$ Tokyo Medical University Hachioji Medical Center, Rheumatology, Tokyo, Japan; ${ }^{2}$ Tokyo Medical University Hachioji Medical Center, Rheumatology, Hachioji, Japan; ${ }^{3}$ Tokyo Rheumatism Pain Clinic, Tokyo, Japan

Background: A recent meta-analysis shows patients with rheumatoid arthritis (RA) are at increased risk of lung and lymphoid malignancies compared with general populations ${ }^{1)}$. Rheumatologists perform cancer screening before treatment of biological disease-modifying anti-rheumatic drugs (DMARDs), and do routine blood, urine and imaging tests to find adverse effects of DMARDs. However, they can't always find malignant solid tumors (cancers) at the early stage in patients with RA.

Objectives: We examine characteristics of cancers in patients with RA and use the data to help diagnose the cancers in early stages during medical checkups.

Methods: In this retrospective study, we studied 397 patients with RA who visited our rheumatology clinic from April 2011 to December 2018. Thirty-one cancers in 29 patients with RA were reviewed. The onset of RA and cancer, the medication situation and prognosis were analyzed.

Results: Twenty-five were female, and 4 males. The mean age of diagnosis of RA and cancer were 55 years old (SD 15.0) and 66 (SD 11.4). The median duration of RA prior to cancer diagnosis was 8 years (IQR 4-18). The median follow-up was 4.3 years (range 0.2-7.8). One case of breast cancer was diagnosed with RA at the same time. Breast, Lung and Gastrointestinal cancers were the most common, followed by uterine cervix and skin. Two female patients had metachronous double cancers (uterine cervix and lung, skin and lung). Sex cancers; uterine cervix 3 , breast 2 , and anal melanoma 1 , were found by cancer screening tests that the patients received voluntarily. Twelve cases were detected by blood tests or imaging tests performed in outpatient settings. Three lung cancers were found by the chest CT at the diagnosis of RA and before treatment of biological DMARDs, which were all at the early stage and cured by the resection. A gastric and a colon cancer, which were detected by worsening of microcytic anemia, were in advanced stage with hepatic metastases. Nineteen patients were treated with methotrexate (MTX) before detection of cancers. But only 4 patients continued MTX after detection of cancers. Six patients were treated with biological DMARDs (TNF inhibitor 3, tocilizumab 2, abatacept 1) before detection of cancers. Two patients were treated with TNF inhibitor until just before the admission for cancer treatment, because the rheumatologists did not notice their patients' cancers. After treatment of cancer, 3 patients were treated with tocilizumab and abatacept. The prognosis of 29 patients were as follows; death by cancers 4 , death by pneumonia 2 , undergoing chemotherapy 6 , in remission 17

Conclusion: The consultation rates for breast and cervix cancer screening are lower in Japan than in European nations. Rheumatologists should encourage their patients to have usual age and sex appropriate cancer screening. A large cohort study showed biological DMARDs did not increase risk of second malignant neoplasm among RA patients with a history of cancer $^{2}$. However, in this study, no patients were treated with TNF inhibitor after treatment of cancer.

\section{REFERENCES}

[1] Simon TA, et al. Incidence of malignancy in adult patients with rheumatoid arthritis: meta-analysis. Arthritis Research and Therapy 2015; 17:212

[2] Dreyer L, et al. Risk of second malignant neoplasm and mortality in patients with rheumatoid arthritis treated with biological DMARDs: a Danish population-based cohort study. Ann Rheum Dis 2017; 0:1-5

Disclosure of Interests: None declared

DOI: 10.1136/annrheumdis-2019-eular.2236

\section{AB0296 \\ ARTERIAL STIFFNESS IN RHEUMATOID ARTHRITIS PATIENTS: DO DISEASE ACTIVITY AND DURATION OF ILLNESS MATTER?}

Awalia Awalia ${ }^{1}$, Satyadi Satyadi ${ }^{2}$, Sony Wibisono ${ }^{3}$, Joewono Soeroso ${ }^{4}$. ${ }^{1}$ Universitas Airlangga/Dr. Soetomo Hospital, Internal Medicine, Division of Rheumatology, Surabaya, Indonesia; ${ }^{2}$ Universitas Airlangga/Dr. Soetomo Hospital, Internal Medicine, Surabaya, Indonesia; ${ }^{3}$ Universitas Airlangga/Dr. Soetomo Hospital, Internal Medicine, Division of Endocrine and Metabolic Diseases, Surabaya, Indonesia; ${ }^{4}$ Universitas Airlangga/Dr. Soetomo Hospital, Internal Medicine, Division of Rheumatology, Surabaya, Indonesia

Background: Recent evidence has demonstrated that the increased risk of mortality in rheumatoid arthritis (RA) patients is largely related to cardiovascular disease (CVD). Overall, RA increases the risk of cardiovascular (CV) mortality by up to $50 \%$ for reasons which are insufficiently understood. Chronic inflammation may impair vascular function and lead to increase of arterial stiffness. Measurement of arterial stiffness provide an independent risk factor of $\mathrm{CV}$ mortality and morbidity. Brachial pulse-wave velocity (BPWV) is a reproducible method to estimate of arterial stiffness. Whether the disease activity or duration of illness has more contribution in arterial stiffness of RA patients is still controversial.

Objectives: To investigate the correlation of disease activity and duration of illness in RA patients with pulse-wave velocity as a measure of arterial stiffness, free of cardiovascular disease and risk factors.

Methods: RA patients aged 55 years-old or younger were screened for the absence of clinical cardiovascular disease or risk factors (diabetes mellitus, dyslipidemia, hypertension, chronic kidney disease, smoking, obesity, cancer, prolong infections, excessive steroid use, or other inflammatory diseases). Patients were subjected to full history taking, clinical examination, and laboratory investigations including serum lipid profile, CRP, and ESR. Then they were recorded for their brachial pulse-wave velocity using PWV Sphygmograph.

Results: There were 30 suitable patients (all were female, mean age was $44.17 \pm 7.98$ years-old, mean duration of illness was $1.88 \pm 0.94$ years). Average systolic blood pressure was $121.67 \pm 7.46 \mathrm{mmHg}$, mean diastolic pressure was $74.00 \pm 4.98 \mathrm{mmHg}$. Average body mass index was $22.43 \pm 1.18$. Mean DAS28-ESR was $3.29 \pm 1.32$, and mean DAS28-CRP was $3.30 \pm 4.02$. Average of BPWV was $14.44 \pm 3.86 \mathrm{~m} / \mathrm{s}$ This study did not show any correlation between duration of illness and arterial stiffness $(p=0.832)$. But it revealed moderate correlation between disease activity (either DAS28-ESR or DAS28-CRP) with arterial stiffness $(r=0.441$ with $p=0.015$ and $r=0.501$ with $p=0.005)$.

Conclusion: This preliminary suggests that disease activity of RA is correlated with arterial stiffness free of cardiovascular disease or risk factors. But duration of illnes does not show any correlation with arterial stiffness as believed before, it may need more sample size to determine that This findings support the importance of inflammation control in RA patients not just to improve quality of life but also to decrease cardiovascular mortality in RA.

\section{REFERENCES}

[1] Klocke R, Cockcroft JR, Taylor GJ, Hall IR, Blake DR. Arterial stiffness and central blood pressure, as determined by pulse wave analysis, in rheumatoid arthritis. Ann Rheum Dis 2003; 62:414-418.

[2] Provan SA, Angel K, Semb AG, Mowinckel P, Agewall S, Star D, Kvien TK. Early prediction of increased arterial stiffness in patients with chronic inflammation: A 15-year followup study of 108 patients with rheumatoid arthritis. The $J$ of Rheumatol 2011; 38:606-612.

[3] Avina-Zubieta JA, Thomas J, Sadatsafavi M, Lehman AJ, Lacaille D. Risk of incident cardiovascular events in patients with rheumatoid arthritis: a meta-analysis of observational studies. Ann Rheum Dis 2012; 71: 15241529.

[4] Youssef G, Allam NT, Gaber W, Afifi A, Hesham D. Increased arterial stiffness in rheumatoid arthritis and Its relation to disease activity: A cross sectional study. The Egyptian Heart J 2018; 70: 35-40.

Disclosure of Interests: None declared

DOI: 10.1136/annrheumdis-2019-eular.3407 